\title{
Aplasia Cutis Congenita (ACC): An Extremely Rare Case in Childhood
}

\author{
Stefan Bittmann*, Elisabeth Luchter, Gloria Villalon and Elena Moschüring-Alieva \\ Department of Pediatrics, Ped Mind Institute, Germany
}

*Correspondence: Stefan Bittmann MD, MA, Department of Pediatrics, Ped Mind Institute, Hindenburgring 448599 Gronau, Germany, Tel: 0049-2565-97325; Fax: 0049-2565-97324; E-mail: stefanbittmann@gmx.de

\begin{abstract}
Aplasia cutis congenita $(\mathrm{ACC})$ is a rare disease characterized by a local absence of skin and, in some cases, subcutaneous tissue. Most cases occur in the scalp, but the lesion can occur anywhere in the trunk and extremities. ACC is usually an isolated defect, but can also be associated with other abnormalities. Most reported cases are sporadic, with a few reports of family events. Neither pathogenesis nor etiology are clarified. In most cases, healing is spontaneous, and apart from keeping the lesion clean, no specific treatment is required. This report presents a case of $\mathrm{ACC}$ at the head and provides a brief review of the literature.
\end{abstract}

Keywords: Aplasia cutis congenita; Child; Head

Received Date: August 25, 2019; Accepted Date: September 20, 2019; Published Date: September 27, 2019

\section{Introduction}

Aplasia cutis congenita (ACC) occurs in 1 of about 10000 newborn babies and is not always reliably detectable due to high variability. The causes are infections, vascular malformations, amniotic defects, teratogenic and genetic causes. A comprehensive theory has not been developed. Vascular factors include diabetes or arterial hypotension of the mother. This can influence circulation in the capillaries of the scalp, which are the finest in the body. Some cases have been influenced to amniotic adhesion, others to teratogenic drugs (antihypertensive ACE inhibitors, the thyrostatic drugs carbimazole and methimazole, valproic acid). Repeated occurrence of isolated ACC in families is rare and may be compatible with autosomal dominant and also, autosomal recessive inheritance. The defect is associated with other abnormalities of the skin, eyes, ear, nose and throat and limbs. Also possible are accompanying malformations of the cardio-vascular and gastrointestinal systems, as well as the urogenital and central nervous systems. ACC can be found as a component of various syndromes: chromosomal abnormalities, especially trisomy 13, Adam's Oliver syndrome, Johanson's Blizzard syndrome and EEC syndrome. Bullous variants of ACC may show histological signs of encephalocele and meningocele, which supports the recent hypothesis that these variants represent the fruste of a neural tube defect. An association is found with omphalocele, gastroschisis and spina bifida. The round or oval skin defects are usually well defined and can occur at the vertex individually or in groups. The size ranges from $1 \mathrm{~cm}^{2}$ to diameters of about $10 \mathrm{~cm}^{2}$. ACC can also manifest itself as a continuous defect of the scalp, cranial bone Citation: Stefan Bittmann, Aplasia Cutis Congenita (ACC): An Extremely Rare Case in Childhood. J Clin Cases Rep 3(3): 66-68. 
and dura. Superficial lesions heal spontaneously with wound care, antibiotics and frequent changes of clothing. Patients with large skin and bone defects are at risk of infection and bleeding. There is no uniform procedure, the measures should be based on the size of the defect and the physical condition of the child. In the case of large defects, early surgical intervention is indicated in order to avoid serious complications such as bleeding and infections. Sufficient skin coverage is sometimes necessary with rotational flaps or further skin grafts.

\section{Case Report}

We report about an 8-year-old girl born with a small defect of $3 \mathrm{~cm}$ in diameter at the head (Figure 1). No corrective operation was performed, nor any other treatment. Family history was clear with no further case in the family or any associated disease. To date, the parents have been sent to a pediatric plastic surgeon to get second opinion. Further corrective operation procedure is planned.

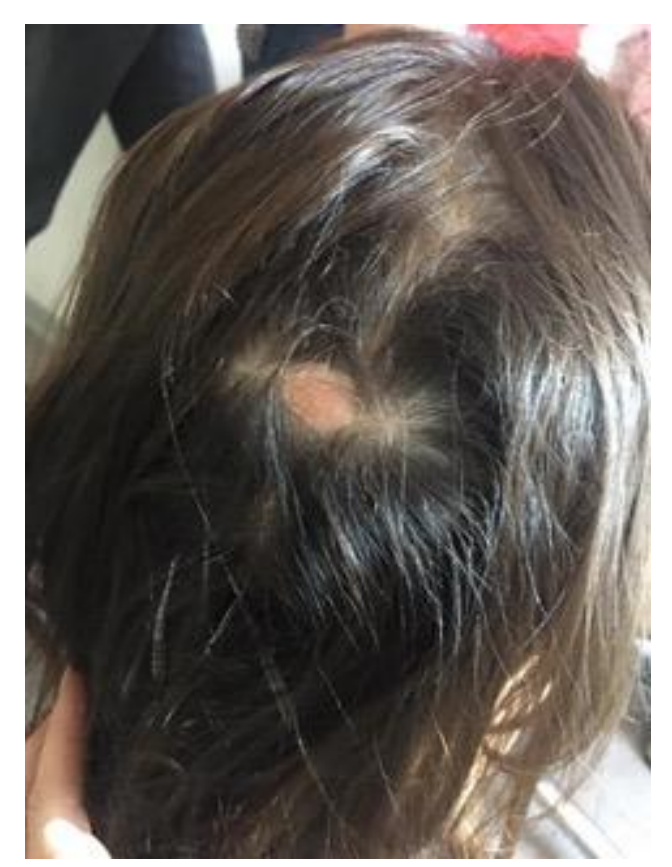

Figure 1: Typical defect of aplasia cutis congenita.

\section{Discussion}

ACC is rarely associated with ulceration in the newborn. ACC is most often found either alone or with EB. Transient bullous dermolysis is a disease, which is a form of dystrophic EB [1]. Intrauterine herpes simplex infections can resemble bullies disease in newborn infants [2]. Neonates with the extreme rare entity of Setleis syndrome may have depressed scarred areas on the temporal scalp resembling healed ACC [3].

Other causes of ulcerations in newborn babies divided from ACC include scalp electrode [4] and pyoderma gangrenosum [5]. The management of ACC is still on a controversial point of view. Most lesions heal with conservative dressing, but large lesions need surgical correction with skin grafts or local skin flaps. Temporary biological dressing to enhance epithelization of the defects are fresh allografts. Cultured epithelial autografts and acellular allogenic dermal grafts can be used together in difficult cases. Flap reconstruction is often the major surgical procedure, with the risk of significant blood loss. The use of cultured keratinocytes is in research and study, but it is still restricted to hospitals having tissue culture laboratory $[6,7]$. 
Conservative outpatient management of the ACC is simple and it obviates the stress of hospital stay and the possibility of infections by resistant hospital borne microorganisms. Secondary wound healing in form of spontaneous healing in our patients did not result in any significant scarring or contractures of near the lesion joints. Slight abnormal hypopigmentation of the healed skin is found regularly.

\section{References}

1. Hanson SG, Fine JD, Levy ML (1999) Three new cases of transient bullous dermolysis of the newborn. Journal of American Academy of Dermatology 40(3): 471-476.

2. Harris HH, Foucar E, Andersen RD, et al. (1986) Intrauterine herpes simplex infection resembling mechanobullous disease in a newborn infant. Journal of American Academy of Dermatology. 15: 114-1155.

3. McGaughran J, Aftimos S (2002) Setleis syndrome: Three new cases and a review of the literature. American Journal of Medical Genetics 111(4): 376-380.

4. Brown ZA, Jung AL, Stenchever MA (1977) Aplasia cutis congenita and the fetal scalp electrode. American Journal of Obstetrics and Gynecology 129: 351-352.

5. Baer MR (1993) Management of unusual presentations of acute leukemia. Hematology/Oncology Clinics of North America 7(1): 275-292.

6. Saraiya HA (2002) Management of aplasia cutis congenita of the scalp: a continuing enigma. British Journal of Plastic Surgery 55: 707-708.

7. Simman R, Priebe CJ, Simon M (2000) Reconstruction of aplasia cutis congenita of the trunk in a newborn infant using acellular allogenic dermal graft and cultured epithelial autografts. Annals of Plastic Surgery 44: 451-454. 